\title{
The study of technological properties of the titanium alloy Ti-6Al-4V. Part 2
}

\author{
R. V. Safiullin ${ }^{1, \dagger}$, M. Kh. Mukhametrakhimov ${ }^{1}$, A. R. Safiullin ${ }^{1}$, S. P. Malysheva ${ }^{1}$, \\ A. N. Kozlov², A. V. Berestov², S. A. Harin', M. A. Morozov ${ }^{3}$ \\ †dr_rvs@mail.ru
}

\begin{abstract}
${ }^{1}$ Institute for Metals Superplasticity Problems of RAS, 39 Khalturin str., Ufa, 450001, Russia ${ }^{2}$ PSC "VSMPO-AVISMA CORPORATION", 1 Parkovaya str., Verkhnaya Salda, 624760, Russia ${ }^{3}$ PSC “ODK-AVIADVIGATEL”, 93 Komsomolskii ave., Perm, 614990, Russia
\end{abstract}

\begin{abstract}
This second part of the article describes the results of studies on technological properties (formability and solid state weldability) of sheet titanium alloy VT6 (Ti-6Al-4V) with improved superplastic properties at low temperatures. When studying the formability of the sheet blanks, the radius of curvature $R$ and average thickness $S$ in the spherical part of the formed samples were determined. The ratio $R / S$ was calculated and represented as a function of the forming time. The presented dependences show that the values of the optimum angle of a conical matrix depend on the forming temperature. The alloy under study had a sufficient formability at temperatures of $T=750$ and $800^{\circ} \mathrm{C}$ corresponding to the temperature range of the low-temperature superplasticity effect. Studies of solid state weldability of sheet blanks made it possible to establish that with an increase in the welding temperature from $T=650$ to $800^{\circ} \mathrm{C}$, the average grain size increased from 1 to $8 \mu \mathrm{m}$, while the relative pore length in the zone of the solid-phase joint decreased from 0.21 at $T=650^{\circ} \mathrm{C}$ to 0.04 at $T=800^{\circ} \mathrm{C}$. The mechanical shear tests of the welded specimens showed that the shear strength of the welded joints varied from 91.4 to $96.9 \%$ of the parent material strength depending on the welding temperature. The study of technological properties revealed that the titanium alloy VT6 could be successfully used in superplastic forming and diffusion welding (SPF/DB) technologies under conditions of lowtemperature superplasticity at the temperatures of $T=700-800^{\circ} \mathrm{C}$. The effective fabrication of a hollow blade model of a threelayer corrugated structure supports this idea.
\end{abstract}

Keywords: sheet titanium alloy, formability, weldability, low-temperature superplasticity, shear strength.

УДК: 669.295:621.785

\section{Исследование технологических свойств титанового сплава Ti-6Al-4V. Часть 2}

\author{
Сафиуллин Р. В. ${ }^{1, \dagger}$, Мухаметрахимов М. Х. ${ }^{1}$, Сафиуллин А. Р. ${ }^{1}$, Малышева С. П. ${ }^{1}$, \\ Козлов А. Н. ${ }^{2}$, Берестов А. В. ${ }^{2}$, Харин С. А. ${ }^{3}$, Морозов М. А. ${ }^{3}$ \\ ${ }^{1}$ Институт проблем сверхпластичности металлов РАН, ул. Халтурина 39, Уфа, 450001, Россия \\ ${ }^{2}$ ПАО «Корпорация ВСМПО-АВИСМА», ул. Парковая 1, В. Салда, 624760, Россия \\ ${ }^{3}$ ПАО «ОДК-Авиадвигатель», Комсомольский пр-т 93, Пермь, 614990, Россия
}

Во второй части статьи описаны результаты исследований технологических свойств (формуемость и свариваемость в твердом состоянии) листового титанового сплава ВT6 (Ti-6Al-4V) с улучшенными сверхпластическими свойствами при пониженных температурах. При проведении исследований формуемости листовых заготовок определяли радиус кривизны $R$ и среднюю толщину $S$ в сферической части отформованных образцов. Было рассчитано отношение $R / S$ и показано в виде зависимостей от времени формовки. Эти зависимости указывают на то, что температура формовки влияет на значения оптимального угла конической матрицы. Исследуемый сплав обладает достаточной формуемостью при температурах $T=750$ и $800^{\circ} \mathrm{C}$, соответствующих температурному интервалу проявления эффекта низкотемпературной сверхпластичности. Исследования свариваемости в твердом состоянии листовых заготовок 
позволили установить, что с увеличением температуры сварки с $T=650$ до $800^{\circ} \mathrm{C}$ увеличивается средний размер зерен с 1 до 8 мкм, при этом относительная протяженность пор в зоне твердофазного соединения уменьшается с 0.21 при $T=650^{\circ} \mathrm{C}$ до 0.04 при $T=800^{\circ} \mathrm{C}$. Механические испытания сварных образцов на срез показали значения прочности сварных соединений в интервале 91.4-96.9\% от прочности основного материала в зависимости от температуры сварки. В результате проведенных исследований технологических свойств установлено, что титановый сплав ВТ6 может быть использован в технологии сверхпластической формовки и диффузионной сварки (СПФ/ДС) в условиях низкотемпературной сверхпластичности при температурах $T=700-800^{\circ} \mathrm{C}$. Подтверждением этого факта является успешное изготовление из этого сплава модели полой лопатки трехслойной гофровой конструкции.

Ключевые слова: листовой титановый сплав, формуемость, свариваемость, низкотемпературная сверхпластичность, прочность на срез.

\section{1. Введение}

Одним из наиболее эффективных технологических процессов обработки листовых материалов, реализующих преимущества сверхпластичности, является сверхпластическая формовка (СПФ), а также ее сочетание с диффузионной сваркой (ДС) [1-4]. Большинство ведущих авиакосмических фирм мира успешно применяют и развивают данную технологию для получения ответственных конструкций летательных аппаратов [5, 6]. Наряду с известными преимуществами процесса СПФ/ДС по сравнению с традиционными технологиями [1-4] у данного метода имеются ограничения и недостатки. Например, высокие рабочие температуры процесса $\left(T=900^{\circ} \mathrm{C}\right)$, требующие использования дорогостоящей жаропрочной оснастки. В случае титановых сплавов высокие температуры ведут к образованию хрупкого альфированного слоя на поверхности получаемых изделий [9-12]. В этой связи снижение температуры обработки является одной из важнейших задач для процесса СПФ/ДС [12 - 19]. В первой части данной работы [19] проведены исследования микроструктуры, механических свойств на растяжение листового сплава ВТ6 с улучшенными свойствами. Показано, что наилучшие сверхпластические свойства наблюдаются в температурном диапазоне $T=700-850^{\circ} \mathrm{C}$. Анизотропия механических свойств в листах отсутствует, это благоприятно для сверхпластической формовки. Целью данной работы является исследование формуемости и свариваемости в твердом состоянии листового титанового сплава ВТ6 с ультрамелкозернистой структурой в условиях низкотемпературной сверхпластичности и возможности использования данного сплава в технологических процессах СПФ/ДС для изготовления облегченных конструкций летательных аппаратов.

\section{2. Материал и методики эксперимента}

В качестве материала исследования был использован лист из титанового сплава ВТ6 (Ti-6Al-4V) толщиной 1 мм производства ПАО «Корпорация ВСМПО-АВИСMA» с улучшенными сверхпластическими свойствами (микроструктура и сверхпластические свойства данного материала исследованы и представлены в 1 части настоящей статьи [19]). Для исследования формуемости листа были выполнены эксперименты, максимально приближенные по схеме напряженно-деформированного состояния материала к реальному технологиче- скому процессу СПФ. Для этого проводили тестовые формовки в коническую матрицу при температурах $T=750$ и $800^{\circ} \mathrm{C}$ при постоянном давлении в течение всего эксперимента. Тестовые формовки были выполнены до степеней деформации $100-150 \%$. При формовке листа в коническую матрицу деформируемая часть заготовки имела форму части сферы, где реализуется напряженное состояние с главными компонентами тензора напряжений $\sigma_{1}=\sigma_{2}=P R / 2 S, \sigma_{3}=0$, где $P$ - давление формующего газа, $R-$ радиус кривизны сферической части образца, $S$ - толщина листа в сферической части образца. Выбором угла раствора а конической матрицы можно добиться постоянства напряжений в формуемой заготовке при постоянном давлении газа, при этом уменьшение радиуса кривизны сферы и ее толщины происходит так, что отношение $R / S$ остается неизменным в ходе всего процесса. Коническая формообразующая матрица имела диаметр входного отверстия 40 мм, а угол раствора конуса составлял $\alpha=58^{\circ}$ в соответствии с рекомендациями работы [14]. Для тестовых формовок использовались образцы в виде пакетов, состоящих из двух сваренных по контуру листовых заготовок, одна из которых была снабжена штуцером.

На Рис. 1 представлены схема сварки давлением листовых заготовок и фотография образца после СПФ. У отформованных образцов определяли форму срединной поверхности в конической и сферической частях, а также измеряли толщину для каждого образца как функцию радиальной координаты. Исследование свариваемости в твердом состоянии листовых заготовок проводили в специальной оснастке в вакуумной печи СНВЭ-1,3.1/16 по схеме, приведенной на Рис. 1а. Давление на соединяемые заготовки передавалось посредством мембраны.

Температура ДС составляла $T=650-800^{\circ} \mathrm{C}$, время сварки $-t=2$ часа, сварку осуществляли в вакууме $P=2.0 \times 10^{-3}$ Па. Из сварных заготовок вырезали образцы для механических испытаний на срез и для исследования микроструктуры. Исследование микроструктуры сварных образцов проводили с помощью сканирующего электронного микроскопа Mira-3 Tescan. Качество сварного соединения оценивали по всей линии соединения. Замеряли размеры пор, подсчитывали их протяженность и определяли относительную долю пор $\left(L_{p}\right)$ в зоне соединения как $L_{p}=\left(L_{p i} / L_{0}\right)$, где $L_{p i}-$ суммарная протяженность пор вдоль линии соединения при длине исследуемого участка $L_{0}$ вдоль линии соединения в плоскости шлифа. 


\section{3. Результаты исследования и их обсуждение}

По результатам исследования механических свойств на растяжение листов титанового сплава ВТ6 установлено [19], что наилучшие сверхпластические свойства наблюдаются при температурах $T=750-800^{\circ} \mathrm{C}$, поэтому исследование формуемости листовых заготовок выполняли при этих же температурах.

На Рис. 2 представлены образцы после формовки при температурах $T=750$ и $800^{\circ} \mathrm{C}$. На Рис. 3 показана форма срединной поверхности в конической и сферической частях отформованных образцов, на Рис. 4 - изменение толщины для каждого образца как функция радиальной координаты. Эти измерения позволили определить радиус кривизны $-R$ и среднюю толщину $S$ образцов в сферической части и рассчитать их отношение, которое представлено в виде зависимостей от времени формовки (Рис. 5).

Результаты, представленные на Рис. 5, показывают связь значения оптимального угла конической матрицы от температуры формовки. Так, при температуре $T=800^{\circ} \mathrm{C}$, отношение $R / S$ растет с увеличением времени формовки, что свидетельствует о необходимости некоторого увеличения угла $\alpha$ для достижения постоянства $R / S$, а при температуре $T=750^{\circ} \mathrm{C}$ отношение $R / S$ уменьшается, и это указывает на необходимость некоторого

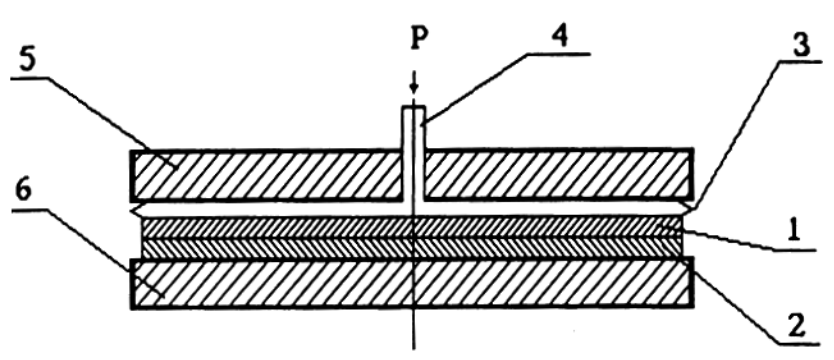

a

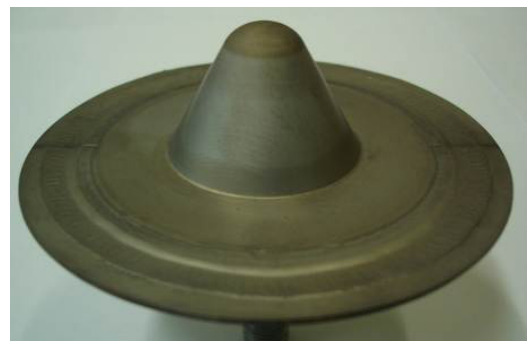

b

Рис. 1. Схема сварки давлением листовых заготовок (1 и 2 - свариваемые заготовки, 3 - мембрана, 4 - трубопровод для подвода рабочего газа, 5 и 6 - силовые плиты) (а) и образец после СПФ (b).

Fig. 1. The scheme for pressure welding of sheet samples ( 1 and 2 - welded samples, 3 - membrane, 4 - the pipeline for a supply of working gas, 5 and 6 - power plates) (a) and the sample after SPF (b).

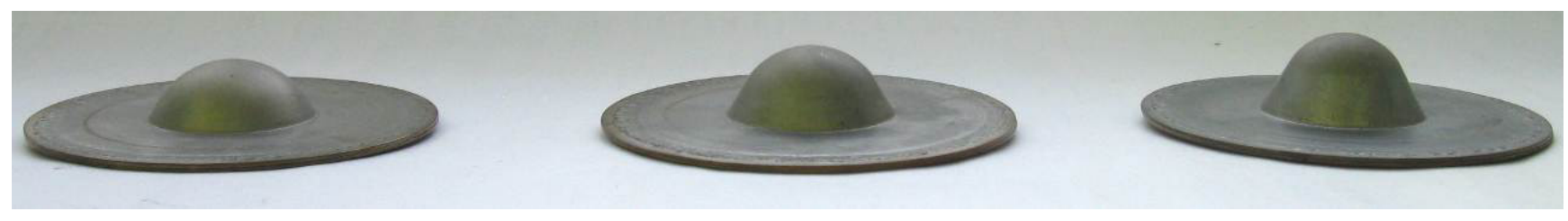

a

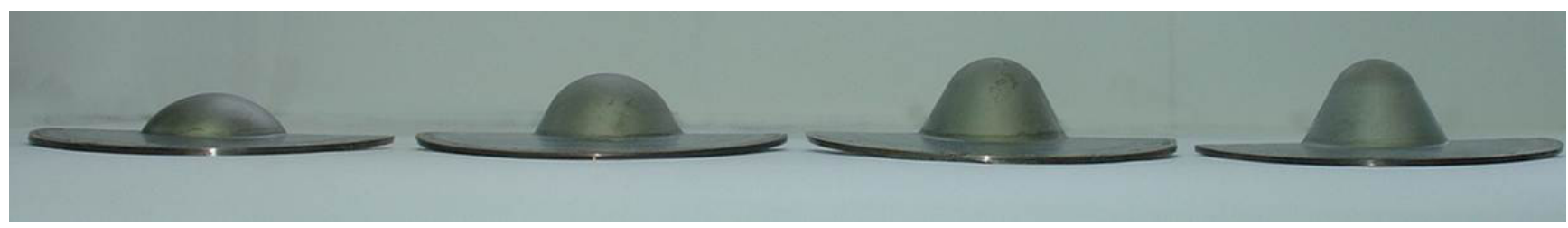

b

Рис. 2. Образцы, отформованные в коническую матрицу: СПФ при $T=750^{\circ} \mathrm{C}(\mathrm{a})$, СПФ при $T=800^{\circ} \mathrm{C}(\mathrm{b})$.

Fig. 2. The samples after $S P F$ in conical matrix: $S P F$ at $T=750^{\circ} \mathrm{C}$ (a), SPF at $T=800^{\circ} \mathrm{C}$ (b).

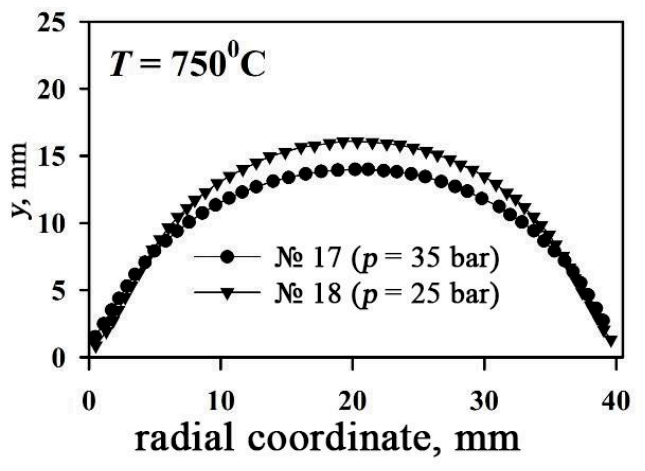

a

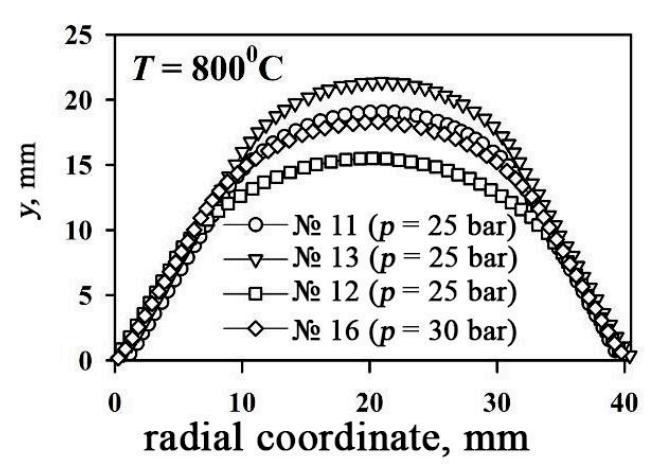

b

Рис. 3. Форма срединной поверхности в конической (a) и сферической (b) частях образцов, отформованных в коническую матрицу.

Fig. 3. The shape of the median surface in the conical (a) and spherical (b) parts of the samples after SPF in conical matrix. 
уменьшения угла $\alpha$. В целом, изменение отношения $R / S$ от времени формовки незначительно для обеих температур $T=750$ и $800^{\circ} \mathrm{C}$ и можно приближенно считать, что действующие напряжения в сферической части образца не зависят от времени. Таким образом, исследуемый сплав обладает достаточной формуемостью при этих температурах.

Исследование свариваемости в твердом состоянии листового сплава ВТ6 с улучшенными свойствами проводили при температурах $T=650-800^{\circ} \mathrm{C}$. Наилучшей свариваемостью сплав в данном состоянии обладает при $T=750-800^{\circ} \mathrm{C}$. На Рис. 6 приведены фотографии микроструктуры твердофазного соединения (ТФС) титанового сплава ВТ6 после сварки при пониженных температурах.

Микроструктурные исследования сварных образцов показали увеличение среднего размера зерен с 1 до 8 мкм при повышении температуры сварки с $T=650$ до $800^{\circ} \mathrm{C}$ Анализ микроструктуры зоны ТФС выявил уменьшение относительной протяженности пор в зоне ТФС с 0.21 (при температуре сварки $T=650^{\circ} \mathrm{C}$ ) до 0.04 (при температуре сварки $\left.T=800^{\circ} \mathrm{C}\right)$.

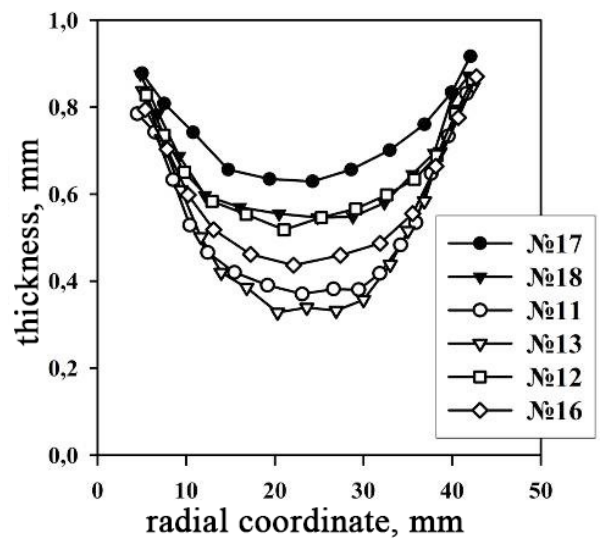

Рис. 4. Изменение толщины образцов, отформованных в коническую матрицу.

Fig. 4. The change in sample thickness formed in a conical matrix.

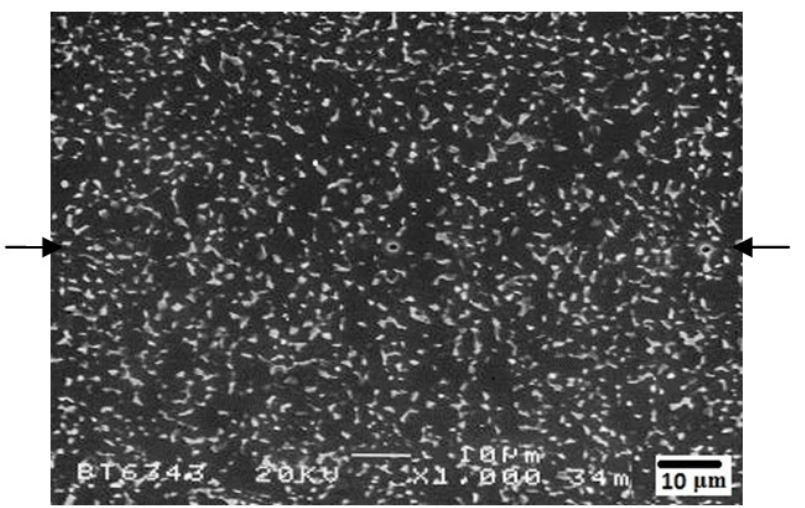

a

Рис. 6. Микроструктура твердофазного соединения титанового сплава ВТ6 с улучшенными сверхпластическими свойствами после диффузионной сварки при температурах $T=750$ (а) и $T=800^{\circ} \mathrm{C}$ (b) (стрелками указана зона сварного соединения).

Fig. 6. The microstructure of the solid-state joints of titanium alloy VT6 with improved superplastic properties after diffusion bonding at temperatures of $T=750$ (a) and $T=800^{\circ} \mathrm{C}$ (b) (arrows indicate bonding zone).
В Табл. 1 приведены результаты механических испытаний сварных образцов на срез. Установлено, что относительная прочность сварных соединений составляет от 91.4 до 96.9\% от прочности основного материала в зависимости от температуры сварки.

Результаты исследований формуемости и свариваемости в твердом состоянии листовых заготовок из титанового сплава ВТ6 сулучшенными свойствами свидетельствуют о возможности их использования для разработки низкотемпературной технологии СПФ/ДС. Оптимальными температурными условиями для процесса СПФ/ДС являются СПФ при $T=700-800^{\circ} \mathrm{C}$ и ДС при $T=800^{\circ} \mathrm{C}$.

С использованием предложенных режимов была разработана опытная технология получения полых трехслойных ячеистых конструкций методом СПФ/ДС, реализована идея низкотемпературной сверхпластичности на практике и получено при температурах $T=750$ и $800^{\circ} \mathrm{C}$ опытное изделие - модель полой лопатки (Рис. 7). Микроструктурные исследования полученных лопаток позволили установить хорошее качество их сварных соединений (Рис. 8).

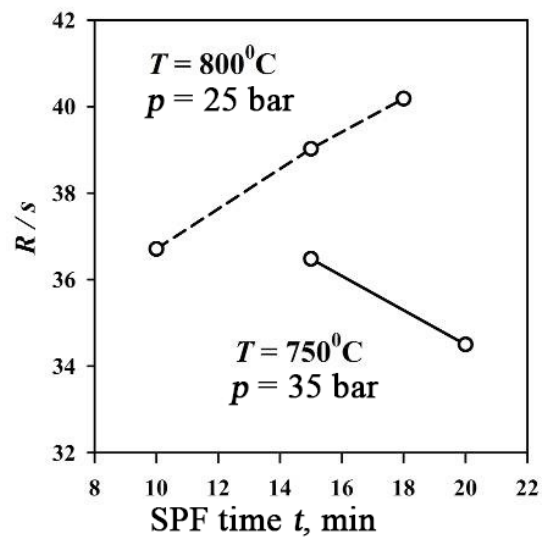

Рис. 5. Зависимости отношения $R / S$ от времени формовки для образцов, изготовленных при $T=750$ и $800^{\circ} \mathrm{C}$.

Fig. 5. The dependences of the $R / S$ ratio on forming time for samples made at $T=750$ and $800^{\circ} \mathrm{C}$.

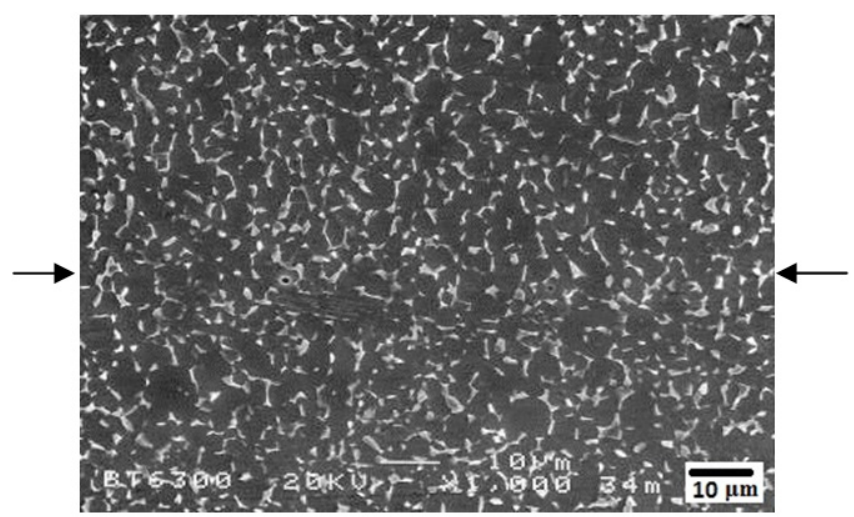

$b$ 
Табл. 1. Зависимость прочности на срез сварных соединений от температуры сварки.

Table 1. Dependence of the shear strength of the welded joints on the welding temperature.

\begin{tabular}{|c|c|c|c|c|c|}
\hline$N$ & $T,{ }^{\circ} \mathrm{C}$ & $P, \mathrm{MPa}$ & $t, \mathrm{~h}$ & $\tau_{\text {cp. }}, \mathrm{MPa}$ & Тип образца / Sample \\
\hline 1 & \multirow{2}{*}{650} & 4 & 2 & $651.8(100 \%)$ & Основной материал / Main part \\
\hline 2 & & 4 & 2 & $631.3(96.9 \%)$ & Сварное соединение / welded joint \\
\hline 3 & \multirow{2}{*}{700} & 4 & 2 & $632.4(100 \%)$ & Основной материал / Main part \\
\hline 4 & & 4 & 2 & $589.2(93.2 \%)$ & Сварное соединение / welded joint \\
\hline 5 & \multirow{2}{*}{750} & 4 & 2 & $615.1(100 \%)$ & Основной материал / Main part \\
\hline 6 & & 4 & 2 & $562.0(91.4 \%)$ & Сварное соединение / welded joint \\
\hline 7 & \multirow{2}{*}{800} & 4 & 2 & $568.8(100 \%)$ & Основной материал / Main part \\
\hline 8 & & 4 & 2 & $551.2(96.9 \%)$ & Сварное соединение / welded joint \\
\hline
\end{tabular}

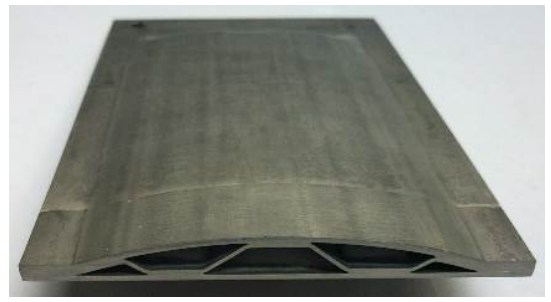

a

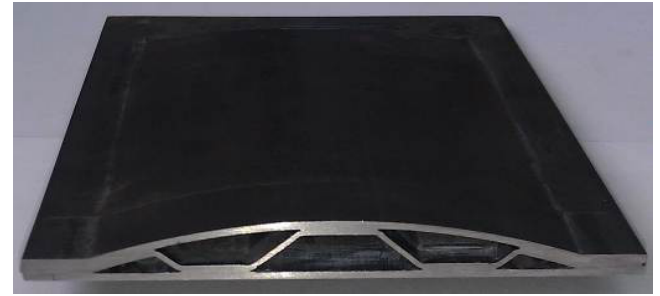

b

Рис. 7. Модели полой лопатки, полученные в условиях низкотемпературной сверхпластичности при $T=750^{\circ} \mathrm{C}(\mathrm{a})$ и $T=800^{\circ} \mathrm{C}(\mathrm{b})$. Fig. 7. Models of a hollow blade, obtained under conditions of low-temperature superplasticity at temperatures of $T=750^{\circ} \mathrm{C}(\mathrm{a})$ and $T=800^{\circ} \mathrm{C}(\mathrm{b})$.

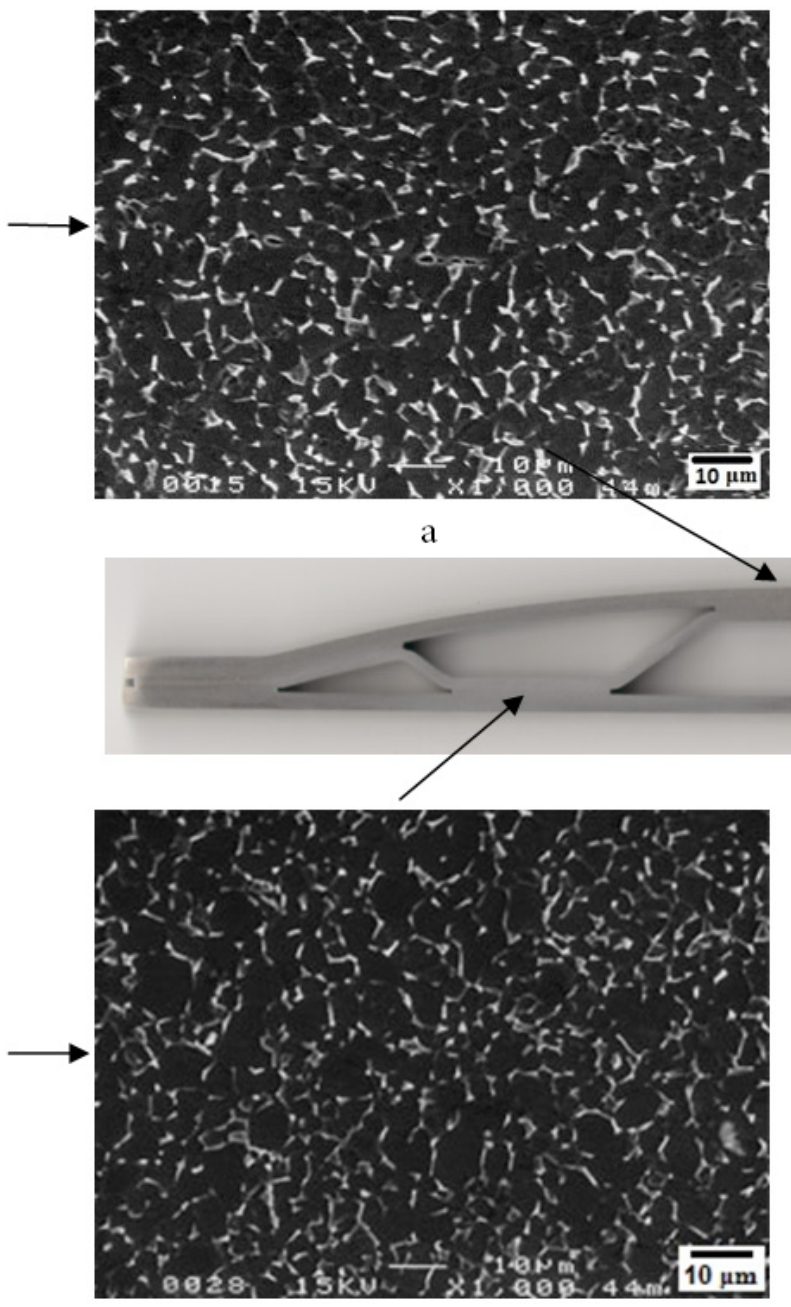

d

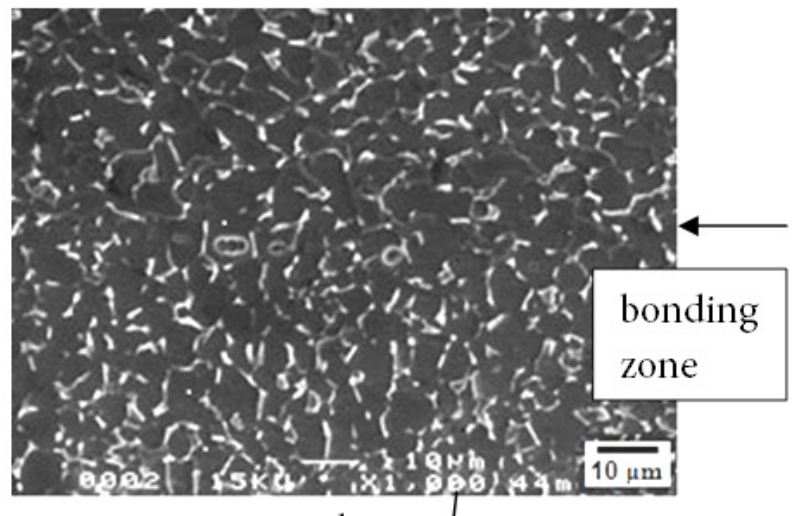

b

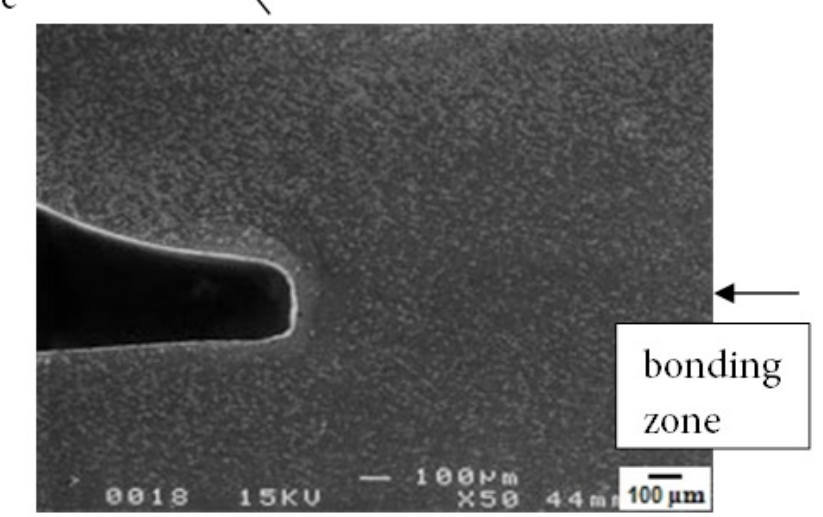

e

Рис. 8. Микроструктура твердофазных соединений модели полой лопатки из сплава ВТ6, полученной при температуре $T=800^{\circ} \mathrm{C}$.

Fig. 8. Microstructure of solid-state joints of hollow blade model made of the alloy VT6 and produced at a temperature of $T=800^{\circ} \mathrm{C}$. 


\section{4. Заключение}

Выполненные комплексные исследования микроструктуры, механических и технологических свойств листового титанового сплава ВТ6 с улучшенными сверхпластическими свойствами производства ПАО «Корпорация ВСМПО-АВИСМА» показали, что листы сплава обладают хорошей формуемостью и свариваемостью при температурах $T=700-800^{\circ} \mathrm{C}$ и могут быть использованы в технологических процессах СПФ/ДС для изготовления облегченных конструкций летательных аппаратов. При температурах $T=750-800^{\circ} \mathrm{C}$ из листов были успешно изготовлены модели полой лопатки трехслойной глфровой конструкции. Исследования полученных лопаток показали хорошее качество полученных в них сварных соединений.

Благодарности/Acknowledgements. Работа выполнена в рамках государственного задания ИПСМ РАН./The present work was accomplished according to the state assignment of IMSP RAS.

\section{Литература/References}

1. E. N. Petrov, V. V. Rodionov, E. N. Kuzmin, R. Ya. Lutfullin, R. V. Safiullin. Honeycomb. Sneginsk (2008) 176 p. (in Russian) [Е.Н. Петров, В.В. Родионов, Э.Н. Кузьмин, Р.Я. Лутфуллин, Р.В. Сафиуллин. Ячеистые конструкции. Снежинск, Изд-во РФЯЦ-ВНИИТФ (2008) $176 \mathrm{c.}$ ]

2. G. B. Stroganov, I. I. Novikov, V. V. Boizov, V. F. Pshirkov. Ispolzovanie sverhplastichnosti $\mathrm{v}$ obrabotke metallov davleniem. Moscow, Mashinostroenie (1989) 108 p. (in Russian) [Строганов Г.Б., Новиков И. И., Бойцов В. В., Пширков В.Ф. Использование сверхпластичности в обработке металлов давлением. Москва, Машиностроение (1989) 132c.]

3. D. Stephen. AGARD Lecture Series. 154(7), 1 (1987).

4. N. Peiton, K. Gamilton. Sverhplasticheskaja formovka konstrukzionnih splavov. Moscow, Metallurgiya (1985) 312 p. (in Russian) [Н. Пейтон, К. Гамильтон Сверхпластическая формовка конструкционных сплавов. Москва, Металлургия (1985) 312 с.]

5. R. V Safiullin. In: Proceedings of 3rd European Conference on Superplastic Forming Euro-SPF 2004. France (2004) P. 167.

6. R. Safiullin. Materials Science Forum. 735, 409 (2013).

7. D. Stephen. In: Proc. Tech. Program International
Conference. USA 2. (1986). P 1087 - 1106.

8. C. H. Hamilton. AGARD Lecture Series. 154(2), 1 (1987).

9. R. V. Safiullin. Letters on Materials. 2(1), 32 (2012). (in Russian) [Р. В. Сафиуллин. Письма о материалах. 2(1), 32 (2012).] DOI: 10.22226/2410-3535-2012-1-32-35

10. R. V. Safiullin. Letters on Materials. 2(1), 36 (2012). (in Russian) [Р. В. Сафиуллин. Письма о материалах. 2(1), 36 (2012).] DOI: 10.22226/2410-3535-2012-1-36-39

11. P.N. Comley. Journal of Materials Engineering and Performance. 17, 183 (2008).

12. D. Sanders, L. Hefti, A. Bryant, S. Zeng, H.Guo. In: Presentation on Euro-SPF-2011 conference. Great Brittan (2011).

13. P. N. Comley. Mater. Sci. Forum. 447 - 448, 233 (2004).

14. O.A. Kaibyshev, R.V. Safiullin, R. Ya. Lutfullin, O.R. Valiakhmetov, R.M. Galeyev, A. Dutta, T. Raghu, G. G. Saha. J. Mat. Sci. and Techn. 22, 343 (2006).

15. O.R. Valiakhmetov, R.M. Galeyev, V.A. Ivanko, R.M. Imayev, A.A. Inozemzev, N.L. Koksharov, A.A. Kruglov, R. Ya. Lutfullin, R.R. Mulykov, R.V. Safiullin, S.A. Harin. Rossiiskie nanotehnologii. 4(11-12), 56 (2009). (in Russian) [О.Р. Валиахметов, Р.М. Галеев, В.А. Иванько, Р.М. Имаев, А.А. Иноземцев, Н.Л. Кокшаров, А.А. Круглов, Р.Я. Лутфуллин, Р.Р. Мулюков, Р.В. Сафиуллин, С.А. Харин. Российские Нанотехнологии. 4(11-12), 56 (2009).]

16. L.D. Hefti. Journal of Materials Engineering and Performance. 17, 178 (2008).

17. B. Swale. In: Proceedings of 3rd European Conference on Superplastic Forming "Euro-SPF 2004". France (2004) P. 39.

18. R. V. Safiullin R.M. Galeyev, M. Kh. Myhametrahimov, R.G. Hazgaliev, S.P. Malysheva, R. R. Mulykov, A.N. Kozlov, A.V. Berestov, M.O. Leder. Titan. 3(53), 47 (2016). (in Russian) [Р. В. Сафиуллин, Р.М. Галеев, М.Х. Мухаметрахимов, Р.Г. Хазгалиев, С.П. Малышева, Р.Р. Мулюков, А.Н. Козлов, А. В. Берестов, М. О. Ледер. Титан. 3(53), 47 (2016).]

19. R. V Safiullin, A. R. Safiullin, S. P. Malysheva, A. N. Kozlov, A. V. Berestov, Galeyev, O.R. Valiakhmetov. Letters on Materials. 6(4), 281 (2016). (in Russian) [Сафиуллин P. B., Сафиуллин А.Р., Малышева С.П., Козлов А.Н., Берестов А.В., Галеев Р.М., Валиахметов О.Р. $\begin{array}{lllll}\text { Письма о материалах. 6(4), } 281 & \text { (2016).] }\end{array}$ DOI: 10.22226/2410-3535-2016-4-281-285

20. R. J. Lederich, S. M. L. Sastry, M. Hayse, T.L. Mackay. Journal of Metals. 8, 16 (1982) 